\title{
NEW RESULTS ON THE CHEBYSHEV POLYNOMIAL BOUNDS FOR CLASSES OF UNIVALENT FUNCTIONS
}

\author{
OYEKAN EZEKIEL ABIODUN*, ADERIBOLE SAMUEL OLAYEMI \\ Department of Mathematical Sciences, Faculty of Science, Olusegun Agagu University of Science and \\ Technology (OAUSTECH), P.M.B. 353, Okitipupa, Ondo State-Nigeria \\ *Corresponding author: shalomfa@yahoo.com, ea.oyekan@oaustech.edu.ng
}

Received May 18, 2020

\begin{abstract}
АвSTRAст. In the present work, by considering a general subclass of univalent functions and using the Chebyshev polynomials, the author determine coefficient bounds for functions in this class. In particular, results, which are presented in this paper, are higher coefficient estimates on the coefficient $\left|a_{4}\right|$ and $\left|a_{5}\right|$ for functions in the function class. Furthermore, we provide the non-linear functional $\left|a_{2} a_{4}-a_{3}^{2}\right|$ for the class.
\end{abstract}

2010 Mathematics Subject Classification. 30A10; 26 D07.

Key words and phrases. Chebyshev polynomials; analytic and univalent functions; coefficient bounds; subordination.

\section{INTRODUCTION}

Let $U=\{z \in c:|z|<1\}$ be the unit disk. In the usual notation, let $A$ denote the class of functions $f(z)$ which are analytic on the unit disk and of the form

$$
f(z)=z+\sum_{n=2}^{\infty} a_{n} z^{n}
$$

Further, by $S$ we shall denote the class of all functions in $A$ which are univalent on $D$. The form (1.1) is the normalized form of functions $f(z) \in A$ for which the normalization condition is given by $f(0)=0$ and $f^{\prime}(0)=1$. Thus,

$$
S=\left\{f \in A: f(0)=f^{\prime}(0)-1=0\right\} .
$$

DOI: 10.28924/APJM/7-24 
If the functions $f$ and $g$ are analytic on $U$, then $f$ is said to be subordinate to $g$, written as

$$
f(z) \prec g(z), \quad(z \in U)
$$

if there exists a Schwartz function $w(z)$ analytic on $U$ with $w(0)=0$ and

$$
w(z)=|z|<1 \quad(z \in U)
$$

such that

$$
f(z)=g(w(z)), \quad(z \in U)
$$

(See details in [1] and [2]). We say that $f(z)$ is convex in domain $U$ if $f: U \rightarrow \mathbb{C}$ is univalent and $f(U)$ is a convex domain with respect to origin. In other words, $f(z)$ is convex if it satisfies the condition

$$
\operatorname{Re}\left(1+\frac{z f^{\prime}(z)}{f(z)}\right)>0, \quad(z \in U)
$$

The class of all functions $f(z)$ satisfying (1.2) is denoted by $K$ such that $f \in K \Leftrightarrow(1.2)$ holds for $z \in U$ where " $\Leftrightarrow$ " implies "if and only if". For each $f \in S$, Fekete and Szegö obtained the sharp bound:

$$
\left|a_{3}-\mu a_{2}^{2}\right| \leq 1+2 e^{-\left(\frac{2 \mu}{1-\mu}\right)}, 0 \leq \mu<1
$$

The functional $\left|a_{3}-\mu a_{2}^{2}\right|$ is well known as the Fekete-Szegö functional. Many other functionals have risen after it, each finding application in certain problems of the geometric functions. Although its origin was in the disproof by Fekete and Szegö of the 1933 conjecture of Littlewood and Paley that the coefficients of odd univalent functions are bounded by unity (see [3]). For $f \in K$ and other related classes of functions, sharp upper bounds for the functional $\left|a_{3}-\mu a_{2}^{2}\right|$ have been studied by various authors (see for example, [4-7]).

\section{Preliminaries and Definitions}

We shall state some basic results and give certain fundamental definitions which are relevant to our main results in order to set a good background for the works in this paper.

Definition 2.1. [4] A function $f \in A$ is said to be in the class $K(\lambda, t), \lambda \geq 0$ and $t \in\left(\frac{1}{2}, 1\right]$, if the following subordinate hold

$$
\left(1-\frac{z f^{\prime}(z)}{f(z)}\right)+\lambda\left(1+\frac{z f^{\prime}(z)}{f(z)}\right) \prec H(z, t):=\frac{1}{1-2 t z+z^{2}}, z \in D
$$


We note that if $t=\cos \alpha, \alpha \in\left(-\frac{\pi}{3}, \frac{\pi}{3}\right)$, then

$$
\begin{aligned}
H(z, t) & =\frac{1}{1-2 t z+z^{2}} \\
& =1+\sum_{n=1}^{\infty} \frac{\sin (n+1) \alpha}{\sin \alpha} z^{n},(z \in D) .
\end{aligned}
$$

Thus

$$
H(z, t)=1+2 \cos \alpha z+\left(3 \cos ^{2} \alpha-\sin ^{2} \alpha\right) z^{2}+\left(4 \cos ^{3} \alpha-4 \cos \alpha \sin \alpha\right) z^{3} \cdots(z \in D)
$$

Following [8], we write

$$
H(z, t)=1+U_{1}(t) z+U_{2}(t) z^{2}+U_{3}(t) z^{3}+\cdots(z \in D, t \in(-1,1))
$$

where $U_{n-1}=\frac{\sin (n \arccos t)}{\sqrt{1-t^{2}}}, n \in \mathbb{N}$ are the Chebyshev polynomials of the second kind. Also, it is known that

$$
U_{n}(t)=2 t U_{n-1}(t)-U_{n-2}(t)
$$

and

$$
\left\{\begin{array}{l}
U_{2}(t)=4 t^{2}-1 \\
U_{3}(t)=8 t^{3}-4 t \\
U_{4}(t)=16 t^{4}-12 t^{2}+1 \\
U_{5}(t)=32 t^{5}-32 t^{4}+6 t \\
U_{6}(t)=64 t^{6}-80 t^{4}+24 t^{2}-1
\end{array}\right.
$$

The Chebyshev polynomials $T_{n}(t), t \in[-1,1]$ of the first kind have the generating function of the form

$$
\sum_{n=0}^{\infty} T_{n}(t) z^{n}=\frac{1-t z}{1-2 t z+z^{2}},(z \in D)
$$

However, the Chebyshev polynomials of the first kind $T_{n}(t)$ and the second kind $U_{n}(t)$ are well connected by the following relationships

$$
\begin{aligned}
\frac{d T_{n}(t)}{d t} & =n U_{n-1}(t), \\
T_{n}(t) & =U_{n}(t)-t U_{n-1}(t), \\
2 T_{n}(t) & =U_{n}(t)-U_{n-2}(t) .
\end{aligned}
$$

For more information on Chebyshev polynomial and their numerous applications, interested reader is referred to Doha [9] and Mason [10]. 
Being motivated by the earlier work of Dzoik et al. [11], Altinkaya and Yakin [4] used the Chebyshev polynomial expansions to provide estimates for the initial coefficient $\left|a_{2}\right|$ and $\left|a_{3}\right|$ of univalent functions in $K(\lambda, t)$ which are stated below:

Theorem 2.1. [4] Let the function $f(z)$ given by (1.1) be in the class $K(\lambda, t)$. Then

$$
\left|a_{2}\right| \leq \frac{2 t}{1+\lambda}
$$

and

$$
\left|a_{3}\right| \leq \frac{\left(2 \lambda^{2}+10 \lambda+4\right) t^{2}}{(1+2 \lambda)(1+\lambda)^{2}}+\frac{t}{1+2 \lambda}-\frac{1}{2(1+2 \lambda)}
$$

However, since the problem of estimating coefficients $\left|a_{n}\right|$ for $n \geq 2$ is still an open problem, the authors in this present work are interested in the higher coefficient bounds for the class $K(\lambda, t)$ which are $\left|a_{4}\right|$ and $\left|a_{5}\right|$. We equally determine the functional $\left|a_{2} a_{4}-a_{3}^{2}\right|$ for the class.

\section{Higher Coefficient Bounds for The Function class $K(\lambda, t)$}

In the section we state and prove the $\left|a_{4}\right|$ and $\left|a_{5}\right|$ as our new results for the function class $K(\lambda, t)$ by making use of the techniques used in [4] and [11]. In order to prove our new results, we shall need the following Lemma:

Lemma 3.1. Let the function $f(z)$ given by (1.1) be in the class $K(\lambda, t)$ then

$$
a_{2}=\frac{2 t C_{1}}{1+\lambda}, a_{3}=\frac{2 t C_{2}}{2(1+2 \lambda)}+\frac{1}{2(1+2 \lambda)}\left\{\left(4 t^{2}-1\right)+\frac{1+3 \lambda}{(1+\lambda)^{2}} 4 t\right\} C_{1}^{2} .
$$

Theorem 3.1. Let the function $f(z)$ given by (1.1) be in the class $K(\lambda, t)$. Then

$$
\begin{aligned}
\left|a_{4}\right| \leq & \frac{\left(16 \lambda^{4}+92 \lambda^{3}+208 \lambda^{2}+100 \lambda+8\right) t^{3}}{3(3 \lambda+1)(2 \lambda+1)(\lambda+1)^{3}}+\frac{\left(16 \lambda^{2}+58 \lambda+14\right) t^{2}}{3(3 \lambda+1)(2 \lambda+1)(\lambda+1)} \\
& +\frac{\left(4 \lambda^{2}+23 \lambda+5\right) t}{3(3 \lambda+1)(2 \lambda+1)(\lambda+1)}-\frac{2}{3(3 \lambda+1)}
\end{aligned}
$$

and

$$
\begin{aligned}
\left|a_{5}\right| \leq & \frac{\left(144 \lambda^{7}+736 \lambda^{6}+2442 \lambda^{5}+3310 \lambda^{4}+1778 \lambda^{4}+478 \lambda^{2}-142 \lambda-82\right) t^{4}}{3(4 \lambda+1)(3 \lambda+1)(2 \lambda+1)^{2}(\lambda+1)^{4}} \\
& +\frac{\left(216 \lambda^{5}+1274 \lambda^{4}+2934 \lambda^{3}+1389 \lambda^{2}+771 \lambda+31\right) t^{3}}{3(4 \lambda+1)(3 \lambda+1)(2 \lambda+1)^{2}(\lambda+1)^{2}} \\
& -\frac{\left(296 \lambda^{4}+1336 \lambda^{3}+444 \lambda^{2}+390 \lambda+8\right) t^{2}}{6(4 \lambda+1)(3 \lambda+1)(2 \lambda+1)^{2}(\lambda+1)}-\frac{16 \lambda^{2}+8 \lambda+3}{8(4 \lambda+1)(2 \lambda+1)^{2}}
\end{aligned}
$$


Proof. Let From (2.1) we have that

$$
\begin{aligned}
& (1-\lambda) \frac{z f^{\prime}(z)}{f(z)}+\lambda\left(1+\frac{z f^{\prime \prime}(z)}{f^{\prime}(z)}\right) \\
& =1+U_{1}(t) w(z)+U_{2}(t) w^{2}(z)+U_{3}(t) w^{3}(z)+U_{4}(t) w^{4}(z)+\cdots,
\end{aligned}
$$

for some analytic function $w$ such that $w(0)=0$ and $|w(z)|<1$ for all $z \in U$.

From the inequalities (2.2) and (2.3), we have that

$$
\begin{aligned}
& (1-\lambda) \frac{z f^{\prime}(z)}{f(z)}+\lambda\left(1+\frac{z f^{\prime \prime}(z)}{f^{\prime}(z)}\right)=1+U_{1}(t) c_{1} z+\left[U_{1}(t) c_{2}\right. \\
& \left.+U_{2}(t) c_{1}^{2}\right] z^{2}+\left[U_{1}(t) c_{3}+2 U_{2}(t) c_{1} c_{2}+U_{3}(t) c_{1}^{3}\right] z^{3}+\left[U_{1}(t) c_{4}\right. \\
& \left.+2 U_{2}(t) c_{1} c_{3}+U_{2}(t) c_{2}^{2}+2 U_{3}(t) c_{1}^{2} c_{2}+U_{4}(t) c_{1}^{4}\right] z^{4}+\cdots
\end{aligned}
$$

It is fairly well-known that if $|w(z)|=\left|c_{1} z+c_{2} z^{2}+c_{3} z^{3}+c_{4} z^{4}+\cdots\right|<1, z \in U$, then

$$
\left|c_{j}\right| \leq 1, \text { for all } j \in \mathbb{N}
$$

and

$$
\left|a_{3}-\mu a_{2}^{2}\right| \leq \max \{1,|\mu|\} \text { for all } \mu \in R
$$

It follows from (3.3) that

$$
\begin{aligned}
& \left(( 1 - \lambda ) z \left(1+4 a_{2} z+6 a_{3} z^{2}+4 a_{2}^{2} z^{2}+8 a_{4} z^{3}+12 a_{2} a_{3} z^{3}\right.\right. \\
& \left.+9 a_{3}^{2} z^{4}+16 a_{2} a_{4} z^{4}+24 a_{3} a_{4} z^{5}+16 a_{4}^{2} z^{6}\right)+\lambda\left(z+3 a_{2} z^{2}\right. \\
& +4 a_{3} z^{3}+5 a_{34} z^{4}+6 a_{2} a_{4} z^{5}+7 a_{3} a_{4} z^{6}+4 a_{4}^{2} z^{7}+2 a_{2}^{2} z^{3} \\
& +5 a_{2} a_{3} z^{4}+3 a_{3}^{2} z^{5}+2 a_{2} z+6 a_{3} z^{2}+12 a_{4} z^{3}+2 a_{2}^{2} z^{2}+8 a_{2} a_{3} z^{3} \\
& \left.\left.+14 a_{2} a_{4} z^{3}+18 a_{3} a_{4} z^{5}+12 a_{4}^{2} z^{6}+6 a_{4}^{2} z^{6}+6 a^{2} a_{3} z^{4}\right)\right) \\
& =\left(z+3 a_{2} z^{2}+4 a_{3} z^{3}+5 a_{4} z^{4}+6 a_{2} a_{4} z^{5}+7 a_{3} a_{4} z^{6}+4 a_{4}^{2} z^{7}\right. \\
& \left.+2 a_{2}^{2} z^{4}+3 a_{3}^{2} z^{5}\right)\left(1+U_{1}(t) c_{1} z+\left[U_{1}(t) c_{2}+U_{2}(t) c_{1}^{2}\right] z^{2}\right. \\
& \left.+\left[U_{1}(t) c_{3}+2 U_{2}(t) c_{1} c_{2}+U_{3}(t) c_{1}^{3}\right] z^{3}+\right)
\end{aligned}
$$

On simplifying (3.6) and equating the coefficient of $z^{4}$ we have

$$
\begin{aligned}
3 a_{4}+9 \lambda a_{4} & =8 a_{3} c_{1}+6 a_{2} c_{2} t+5 a_{2} a_{3}-2 c_{1} c_{2}+2 c_{3} t-3 a_{2} c_{1}^{2} \\
& +4 a_{2}^{2} c_{1} t-4 c_{1}^{3} t+8 c_{1} c_{2} t^{2}+12 a_{2} c_{1}^{2} t^{2} \\
& +8 c_{1}^{3} t^{3}-a_{2} a_{3} \lambda-12 a_{2} a_{3} .
\end{aligned}
$$


Substituting (3.1) into (3.7) we have,

$$
\begin{gathered}
a_{4}=\frac{8 c_{1}^{3} t^{3}}{3(3 \lambda+1)}+\frac{16 c_{1}^{3} t^{3}}{3(3 \lambda+1)(2 \lambda+1)}+\frac{8 c_{1}^{3} t^{3}}{3(3 \lambda+1)(\lambda+1)} \\
+\frac{4 \lambda c_{1}^{3} t^{3}}{3(3 \lambda+1)(2 \lambda+1)(\lambda+1)}-\frac{4 c_{1}^{3} t}{3(3 \lambda+1)}+\frac{8 c_{1} c_{2} t^{2}}{3(3 \lambda+1)} \\
+\frac{16 c_{1}^{3} t^{3}}{3(3 \lambda+1)(\lambda+1)^{2}}+\frac{20 c_{1}^{3} t^{3}}{3(3 \lambda+1)(2 \lambda+1)(\lambda+1)} \\
+\frac{4 \lambda c_{1}^{3} t^{3}}{(3 \lambda+1)(2 \lambda+1)(\lambda+1)^{2}}-\frac{4 c_{1}^{3} t}{3(3 \lambda+1)(2 \lambda+1)}-\frac{2 c_{1}^{3} t}{(3 \lambda+1)(\lambda+1)} \\
-\frac{\lambda c_{1}^{3} t}{3(3 \lambda+1)(2 \lambda+1)}+\frac{4 c_{1} c_{2} t^{2}}{3(3 \lambda+1)(2 \lambda+1)}+\frac{116 c_{1}^{3} t^{3}}{(3 \lambda+1)(\lambda+1)} \\
+\frac{2 \lambda c_{1} c_{2} t^{2}}{3(3 \lambda+1)(2 \lambda+1)(\lambda+1)}-\frac{8 \lambda(3 \lambda+1)(2 \lambda+1)(\lambda+1)}{3\left(\lambda c_{1}^{3} t^{3}\right.} \\
-\frac{7 c_{1}^{3} t}{3(3 \lambda+1)(2 \lambda+1)(\lambda+1)^{3}}-\frac{2 c_{1} c_{2}}{3(3 \lambda+1)} \\
-\frac{56 c_{1}^{3} t^{3}}{3(3 \lambda+1)(2 \lambda+1)(\lambda+1)}+\frac{2 c_{3} t}{3(3 \lambda+1)}-\frac{14 c_{1} c_{2} t^{2}}{3(3 \lambda+1)(2 \lambda+1)(\lambda+1)} \\
+\frac{2}{3(3 \lambda+1)(2 \lambda+1)(\lambda+1)^{3}}
\end{gathered}
$$

By applying (3.4) to (3.8) and then simplify, we get

$$
\begin{aligned}
& \left|a_{4}\right| \leq \frac{\left(16 \lambda^{4}+92 \lambda^{3}+208 \lambda^{2}+100 \lambda+8\right) t^{3}}{3(3 \lambda+1)(2 \lambda+1)(\lambda+1)^{3}} \\
& +\frac{\left(16 \lambda^{2}+58 \lambda+14\right) t^{2}}{3(3 \lambda+1)(2 \lambda+1)(\lambda+1)^{3}} \\
& +\frac{\left(4 \lambda^{2}+23 \lambda+5\right) t}{3(3 \lambda+1)(2 \lambda+1)(\lambda+1)^{3}}-\frac{2}{3(3 \lambda+1)}
\end{aligned}
$$

Next, in order to fine bound on $\left|a_{5}\right|$, we simplify (3.6) and take the coefficient $z^{5}$ such that

$$
\begin{aligned}
& a_{5}(4 \lambda+1)=3 a_{3}^{2}+6 a_{2} a_{4}-c_{2}^{2}-2 c_{1} c_{3}+2 c_{4} t+6 a_{2} c_{3} t \\
& +8 a_{3} c_{2} t+10 a_{4} c_{1} t-4 a_{3} c_{1}^{2}-6 a_{2} c_{1} c_{2}+8 c_{1} c_{3} t^{2}-2 a_{2}^{2} c_{1}^{2} \\
& +c_{1}^{4}+10 a_{2} a_{3} c_{1} t+4 a_{2}^{2} c_{2} t-12 c_{1}^{2} c_{2} t+4 c_{2}^{2} t^{2}+24 a_{2} c_{1} c_{2} t^{2} \\
& +16 a_{3} c_{1}^{2} t^{2}-12 a_{2} c_{1}^{3}+8 a_{2}^{2} c_{1}^{2} t^{2}-12 c_{1}^{4} t^{2}+24 c_{1}^{2} c_{2} t^{3} \\
& +16 c_{1}^{4} t^{4}-16 a_{2} a_{4}+9 a_{3}^{2}+4 a_{2} a_{4} \lambda .
\end{aligned}
$$


By substituting $a_{2}, a_{3}$ and $a_{4}$ into (3.10) we have,

$$
\begin{aligned}
& a_{5}=\frac{4 c_{1}^{4} t^{4}}{4 \lambda+1}+\frac{20 c_{1}^{4} t^{4}}{3(4 \lambda+1)(3 \lambda+1)}+\frac{8 c_{1}^{4} t^{4}}{(4 \lambda+1)(2 \lambda+1)} \\
& -\frac{16 \lambda c_{1}^{4} t^{4}}{3(4 \lambda+1)(3 \lambda+1)(\lambda+1)}-\frac{3 c_{1}^{4} t^{2}}{4 \lambda+1}+\frac{6 c_{1}^{2} c_{2} t^{3}}{4 \lambda+1} \\
& -\frac{6 c_{1}^{4} t^{4}}{(4 \lambda+1)(2 \lambda+1)^{2}}-\frac{40 c_{1}^{4} t^{4}}{3(4 \lambda+1)(3 \lambda+1)(\lambda+1)}-\frac{8 c_{1}^{4} t^{4}}{(4 \lambda+1)(\lambda+1)^{2}} \\
& +\frac{34 c_{1}^{4} t^{4}}{(4 \lambda+1)(2 \lambda+1)(\lambda+1)}-\frac{16 \lambda c_{1}^{4} t^{4}}{(4 \lambda+1)(3 \lambda+1)(\lambda+1)^{2}} \\
& -\frac{22 \lambda c_{1}^{4} t^{4}}{3(4 \lambda+1)(3 \lambda+1)(2 \lambda+1)(\lambda+1)}-\frac{8 \lambda^{2} c_{1}^{4} t^{4}}{3(4 \lambda+1)(3 \lambda+1)(2 \lambda+1)(\lambda+1)^{2}} \\
& -\frac{10 c_{1}^{4} t^{4}}{3(4 \lambda+1)(3 \lambda+1)}-\frac{4 c_{1}^{4} t^{4}}{(4 \lambda+1)(2 \lambda+1)}-\frac{6 c_{1}^{4} t^{4}}{(4 \lambda+1)(\lambda+1)} \\
& +\frac{8 \lambda c_{1}^{4} t^{4}}{3(4 \lambda+1)(3 \lambda+1)(\lambda+1)}+\frac{20 c_{1}^{2} c_{2} t^{3}}{3(4 \lambda+1)(3 \lambda+1)}+\frac{8 c_{1}^{2} c_{2} t^{3}}{(4 \lambda+1)(2 \lambda+1)} \\
& +\frac{12 c_{1}^{2} c_{2} t^{3}}{(4 \lambda+1)(\lambda+1)}-\frac{16 \lambda c_{1}^{2} c_{2} t^{3}}{3(4 \lambda+1)(3 \lambda+1)(\lambda+1)}-\frac{80 c_{1}^{4} t^{4}}{3(4 \lambda+1)(3 \lambda+1)(\lambda+1)^{2}} \\
& +\frac{14 c_{1}^{4} t^{4}}{(4 \lambda+1)(2 \lambda+1)(\lambda+1)^{2}}-\frac{36 c_{1}^{4} t^{4}}{(4 \lambda+1)(\lambda+1)(2 \lambda+1)^{2}} \\
& -\frac{10 c_{1}^{4} t^{4}}{(4 \lambda+1)(3 \lambda+1)(2 \lambda+1)(\lambda+1)}-\frac{32 \lambda c_{1}^{4} t^{4}}{3(4 \lambda+1)(3 \lambda+1)(\lambda+1)^{3}} \\
& -\frac{10 \lambda c_{1}^{4} t^{4}}{(4 \lambda+1)(3 \lambda+1)(2 \lambda+1)(\lambda+1)^{2}}-\frac{8 \lambda^{2} c_{1}^{4} t^{4}}{3(4 \lambda+1)(3 \lambda+1)(\lambda+1)^{3}}+\frac{c_{1}^{4}}{4(4 \lambda+1)} \\
& -\frac{3 c_{1}^{2} c_{2} t}{4 \lambda+1}+\frac{3 c_{1}^{4} t^{2}}{(4 \lambda+1)(2 \lambda+1)^{2}}-\frac{10 c_{1}^{4} t^{2}}{3(4 \lambda+1)(3 \lambda+1)(2 \lambda+1)}-\frac{2 c_{1}^{4} t^{2}}{(4 \lambda+1)(\lambda+1)^{2}} \\
& +\frac{5 c_{1}^{4} t^{2}}{3(4 \lambda+1)(3 \lambda+1)(\lambda+1)}-\frac{17 c_{1}^{4} t^{2}}{2(4 \lambda+1)(3 \lambda+1)(\lambda+1)} \\
& +\frac{4 \lambda c_{1}^{4} t^{2}}{(4 \lambda+1)(3 \lambda+1)(\lambda+1)^{2}}+\frac{11 \lambda c_{1}^{4} t^{2}}{6(4 \lambda+1)(3 \lambda+1)(2 \lambda+1)(\lambda+1)} \\
& +\frac{2 \lambda^{2} c_{1}^{4} t^{2}}{3(4 \lambda+1)(3 \lambda+1)(2 \lambda+1)(\lambda+1)^{2}}+\frac{c_{2}^{2} t^{2}}{(4 \lambda+1)}+\frac{2 c_{1} c_{2} t^{2}}{(4 \lambda+1)} \\
& -\frac{6 c_{1}^{2} c_{2} t^{3}}{(4 \lambda+1)(\lambda+1)^{2}}+\frac{20 c_{1}^{2} c_{2} t^{3}}{3(4 \lambda+1)(3 \lambda+1)(2 \lambda+1)}+\frac{4 c_{1}^{2} c_{2} t^{3}}{(4 \lambda+1)(\lambda+1)^{2}} \\
& -\frac{10 c_{1}^{2} c_{2} t^{3}}{3(4 \lambda+1)(3 \lambda+1)(\lambda+1)}+\frac{17 c_{1}^{2} c_{2} t^{3}}{(4 \lambda+1)(2 \lambda+1)(\lambda+1)}-\frac{8 \lambda c_{1}^{2} c_{2} t^{3}}{(4 \lambda+1)(3 \lambda+1)(\lambda+1)^{2}} \\
& -\frac{11 \lambda c_{1}^{2} c_{2} t^{3}}{3(4 \lambda+1)(3 \lambda+1)(2 \lambda+1)(\lambda+1)}-\frac{4 \lambda^{2} c_{1}^{2} c_{2} t^{3}}{3(4 \lambda+1)(3 \lambda+1)(2 \lambda+1)(\lambda+1)^{2}} \\
& -\frac{80 c_{1}^{4} t^{4}}{3(4 \lambda+1)(3 \lambda+1)(\lambda+1)^{3}}-\frac{20 c_{1}^{4} t^{4}}{(4 \lambda+1)(2 \lambda+1)(\lambda+1)^{3}}
\end{aligned}
$$




$$
\begin{aligned}
& -\frac{30 c_{1}^{4} t^{4}}{(4 \lambda+1)(2 \lambda+1)^{2}(\lambda+1)^{2}}-\frac{130 c_{1}^{4} t^{4}}{3(4 \lambda+1)(3 \lambda+1)(2 \lambda+1)(\lambda+1)^{2}} \\
& +\frac{152 \lambda c_{1}^{4} t^{4}}{3(4 \lambda+1)(3 \lambda+1)(2 \lambda+1)(\lambda+1)^{3}}+\frac{16 \lambda^{2} c_{1}^{4} t^{4}}{3(4 \lambda+1)(3 \lambda+1)(2 \lambda+1)(\lambda+1)^{4}} \\
& +\frac{c_{1}^{4}}{2(4 \lambda+1)(2 \lambda+1)}-\frac{5 c_{1}^{2} c_{2} t}{3(4 \lambda+1)(3 \lambda+1)}-\frac{2 c_{1}^{2} c_{2} t}{(4 \lambda+1)(2 \lambda+1)}-\frac{3 c_{1}^{2} c_{2} t}{(4 \lambda+1)(\lambda+1)} \\
& +\frac{4 \lambda c_{1}^{2} c_{2} t}{3(4 \lambda+1)(3 \lambda+1)(\lambda+1)}+\frac{10 c_{1}^{4} t^{2}}{(4 \lambda+1)(3 \lambda+1)(\lambda+1)^{2}} \\
& +\frac{4 c_{1}^{4} t^{2}}{(4 \lambda+1)(2 \lambda+1)(\lambda+1)^{2}}+\frac{9 c_{1}^{4} t^{2}}{(4 \lambda+1)(2 \lambda+1)^{2}(\lambda+1)} \\
& +\frac{25 c_{1}^{4} t^{2}}{2(4 \lambda+1)(3 \lambda+1)(2 \lambda+1)(\lambda+1)}-\frac{3 \lambda c_{1}^{4} t^{2}}{3(4 \lambda+1)(3 \lambda+1)(2 \lambda+1)(\lambda+1)^{2}} \\
& +\frac{2 c_{2}^{2} t^{2}}{(4 \lambda+1)(2 \lambda+1)}+\frac{5 c_{1} c_{3} t^{2}}{3(4 \lambda+1)(3 \lambda+1)}+\frac{3 c_{1} c_{3} t^{2}}{(4 \lambda+1)(\lambda+1)^{3}} \\
& -\frac{4 \lambda c_{1} c_{3} t^{2}}{3(4 \lambda+1)(3 \lambda+1)(\lambda+1)}-\frac{20 c_{1}^{2} c_{2} t^{3}}{(4 \lambda+1)(3 \lambda+1)(\lambda+1)^{2}} \\
& -\frac{8 c_{1}^{2} c_{2} t^{3}}{(4 \lambda+1)(2 \lambda+1)(\lambda+1)^{2}}-\frac{18 c_{1}^{2} c_{2} t^{3}}{(4 \lambda+1)(2 \lambda+1)^{2}(\lambda+1)} \\
& -\frac{25 c_{1}^{2} c_{2} t^{3}}{(4 \lambda+1)(3 \lambda+1)(2 \lambda+1)(\lambda+1)}+\frac{6 \lambda c_{1}^{2} c_{2} t^{3}}{3(4 \lambda+1)(3 \lambda+1)(2 \lambda+1)(\lambda+1)^{2}} \\
& +\frac{74 c_{1}^{4} t^{4}}{(4 \lambda+1)(2 \lambda+1)^{2}(\lambda+1)^{3}}+\frac{240 c_{1}^{4} t^{4}}{3(4 \lambda+1)(3 \lambda+1)(2 \lambda+1)(\lambda+1)^{3}} \\
& -\frac{24 \lambda c_{1}^{4} t^{4}}{(4 \lambda+1)(3 \lambda+1)(2 \lambda+1)(\lambda+1)^{4}}-\frac{3 c_{1}^{4}}{8(4 \lambda+1)(2 \lambda+1)^{2}}-\frac{c_{2}^{2}}{4(4 \lambda+1)} \\
& -\frac{c_{1} c_{3}}{2(4 \lambda+1)}+\frac{3 c_{1}^{2} c_{2} t}{2(4 \lambda+1)(2 \lambda+1)^{2}}+\frac{10 c_{1}^{2} c_{2} t}{3(4 \lambda+1)(3 \lambda+1)(\lambda+1)}+\frac{c_{4} t}{2(4 \lambda+1)} \\
& -\frac{6 c_{1}^{4} t^{2}}{(4 \lambda+1)(2 \lambda+1)^{2}(\lambda+1)^{2}}-\frac{35 c_{1}^{4} t^{2}}{3(4 \lambda+1)(3 \lambda+1)(2 \lambda+1)(\lambda+1)^{2}} \\
& -\frac{3 c_{2}^{2} t^{2}}{2(4 \lambda+1)(2 \lambda+1)^{2}}-\frac{10 c_{1} c_{3} t^{2}}{3(4 \lambda+1)(3 \lambda+1)(\lambda+1)}+\frac{12 c_{1}^{2} c_{2} t^{3}}{(4 \lambda+1)(2 \lambda+1)^{2}(\lambda+1)^{2}} \\
& +\frac{70 c_{1}^{2} c_{2} t^{3}}{3(4 \lambda+1)(3 \lambda+1)(2 \lambda+1)(\lambda+1)^{2}}-\frac{24 c_{1}^{4} t^{4}}{(4 \lambda+1)(2 \lambda+1)^{2}(\lambda+1)^{4}} \\
& -\frac{280 c_{1}^{4} t^{4}}{3(4 \lambda+1)(3 \lambda+1)(2 \lambda+1)(\lambda+1)^{4}} \text {. }
\end{aligned}
$$


By applying (3.4) to (3.11) and then simplify, we get

$$
\begin{aligned}
\left|a_{5}\right| & \leq \frac{A t^{4}}{3(4 \lambda+1)(3 \lambda+1)(2 \lambda+1)^{2}(\lambda+1)^{4}} \\
& +\frac{\left(216 \lambda^{5}+1274 \lambda^{4}+2934 \lambda^{3}+1389 \lambda^{2}+771 \lambda+31\right) t^{3}}{3(4 \lambda+1)(3 \lambda+1)(2 \lambda+1)^{2}(\lambda+1)^{4}} \\
& -\frac{\left(296 \lambda^{4}+1336 \lambda^{3}+444 \lambda^{2}+390 \lambda+8\right) t^{2}}{6(4 \lambda+1)(3 \lambda+1)(2 \lambda+1)^{2}(\lambda+1)^{2}} \\
& -\frac{\left(180 \lambda^{4}+816 \lambda^{3}+606 \lambda^{2}+235 \lambda+26\right) t}{6(4 \lambda+1)(3 \lambda+1)(2 \lambda+1)^{2}(\lambda+1)} \\
& -\frac{16 \lambda^{2}+8 \lambda+3}{8(4 \lambda+1)(2 \lambda+1)^{2}}
\end{aligned}
$$

where

$$
A:=144 \lambda^{7}+736 \lambda^{6}+2442 \lambda^{5}+3310 \lambda^{4}+1778 \lambda^{3}+478 \lambda^{2}-142 \lambda-82 .
$$

Which is the end of the proof.

\section{FeKete-Szegö inequalities for the function Class $K(\lambda, t)$.}

Theorem 4.1. Let $f$ given by (1.1) be in the class $K(\lambda, t)$, then

$$
\begin{aligned}
\left|a_{2} a_{4}-a_{3}^{2}\right| & \leq\left\{\left(\frac { 1 + 2 \lambda } { 3 ( 1 + \lambda ) ^ { 4 } [ ( 1 + 2 \lambda ) ( 1 + 3 \lambda ) ] ^ { 2 } } \left[(1+\lambda)^{3}(1+3 \lambda)(23-\lambda)\right.\right.\right. \\
& -8(\lambda)^{2}(1+3 \lambda)(55 \lambda+22)+8 \lambda(1+2 \lambda) \\
& \left.+4(1+\lambda)(1+3 \lambda)(33+16 \lambda)] t^{4}+t^{5}+\frac{4[(1+2 \lambda)(5+\lambda)+(5 \lambda-3)]}{(1+\lambda)^{2}(1+2 \lambda)(1+3 \lambda)} t^{3}\right) \\
& -\left(\frac { 1 } { 3 ( 1 + \lambda ) ^ { 4 } [ ( 1 + 2 \lambda ) ( 1 + 3 \lambda ) ] ^ { 2 } } \left[13(1+2 \lambda)^{2}(1+3 \lambda)\right.\right. \\
& \left.+(1+3 \lambda)^{2}(95-72 \lambda)\right] t^{4}+\frac{(1+\lambda)^{2}(1+2 \lambda)^{2}\left(4 t^{5}+t^{3}\right)}{[(1+\lambda)(1+2 \lambda)]^{2}} \\
& \left.\left.+\frac{t^{3}}{2(1+\lambda)^{2}(1+2 \lambda)^{4}}+\frac{2\left(\lambda^{2}+5 \lambda+4\right)}{[(1+\lambda)(1+2 \lambda)]^{2}} t^{2}+\frac{4}{3(1+\lambda)(1+3 \lambda)} t\right)\right\}
\end{aligned}
$$

Taking $\lambda=1$ in the above theorems we get the following corollary.

Corollary 4.1. If $f \in K(t)$, then

$$
\begin{gathered}
\left|a_{4}\right| \leq \frac{3}{4} t^{3}+\frac{11}{9} t^{2}+\frac{4}{9} t-\frac{1}{6} \\
\left|a_{5}\right| \leq \frac{3733}{4320} t^{4}+\frac{441}{144} t^{3}-\frac{1237}{2160} t^{2}-\frac{621}{1440} t-\frac{3}{40} \\
\left|a_{2} a_{4}-a_{3}^{2}\right| \leq \frac{71}{72} t^{2}+\frac{1297}{648} t^{3}-\frac{5639}{1728} t^{4}-7 t^{5}-\frac{1}{6} t .
\end{gathered}
$$




\section{Conclusion}

The higher coefficients bounds established for the class $K(\lambda, t)$ in this work were not easily trackable. Some aspect of the computations were achieved through Python Programming Software and painstaken manual computation.

\section{REFERENCES}

[1] P. L. Duren, Grundlehen der Mathematischen Wissencheften, Springer, New York, NY, USA, 1983.

[2] H. M. Strivastava and S. Owa, Current Topics In Analytic Function Theory,World Scientific Publishing Company, Singapore, New Jersey, London and Hong Kong, 1992.

[3] M. Fekete and G. Szeg, Eine bemerkang ber Ungerade Schlichte funktionen, J. Lond. Math. Soc. 2 (1933), 85-89.

[4] S. Altinkaya and S. Yalcin, On the Chebyshev Polynomial Bounds for Classes of Univalent Functions, Khayyam J. Math. 2 (2016), no 1, 1-5.

[5] F. R. keogh and E. P. Merkes, A Coefficient Inequality for Certain Classes of Analytic Functions, Pro. Amer. Math. Soc. 20 (1969), 8-12.

[6] M. H. Al-Abbadi and Darus M., Fekete-Szeg Theorem for a Certain Class of Analytic Functions, Sains Malaysiana, 40 (2011), no 4, 385-389.

[7] O. A. Olukoya and E. A. Oyekan, Coefficient Bounds for the functions in the Class of Modified Hyperbolic Tanget Function, Coast J. Faculty of Sci. 1 (2020), no 2, 222-224.

[8] E.T. Whittaker and G.N. Watson, A Course of Modern Analysis: An Introduction to the General Theory of Infinite Processes of Analytic Functions; with an Account of the Principal Transcendental Functions, 4th ed., Cambridge University Press, 1963.

[9] E. H. Doha. The First and Second Kind Chebyshev Coefficient of Moments of the General-order of an Infinitely Differentiable Function, Int. J. Comput. Math. 51(1994), 21-35.

[10] J. C. Mason, Chebyshev Polynomials Approximations for the L-membrane Eigenvalue Problems, SIAM J. Appl. Math. 15 (1967), 172-186.

[11] J. Dziok, R. K. Raina and J. Sokol, Applications of Chebyshev Polynomials for Classes of Analytic Functions, C.R. Acad. Sci. Paris, Soc. I, 353 (2015), 433-438. 